\title{
(Epi)Genetic Analyses of Age-Related Macular Degeneration: Case-Control and Discordant Twin Studies
}

\author{
John N. Hutchinson ${ }^{a}$ Jes Fagerness ${ }^{a}$ Andrew Kirby ${ }^{a}$ Robyn Reynolds ${ }^{b}$ \\ Alex Zak ${ }^{c}$ Alexander Gimelbrant ${ }^{c, d}$ Robert Plenge ${ }^{e, f}$ Mark Daly ${ }^{a}$ \\ Andrew Chess ${ }^{a, g}$ Johanna M. Seddon ${ }^{b, h, i}$
}

\begin{abstract}
${ }^{a}$ Center for Human Genetic Research, Massachusetts General Hospital, ${ }^{b}$ Ophthalmic Epidemiology and Genetics Service, New England Eye Center, Tufts Medical Center, Boston, Mass., ' Department of Cancer Biology, Dana-Farber Cancer Institute, ${ }^{d}$ Department of Genetics, Harvard Medical School, e Rheumatology and Immunology Department, Brigham and Women's Hospital, ${ }^{f}$ Harvard Medical School, Harvard University, Boston, Mass., ${ }^{9}$ Department of Developmental and Regenerative Biology, Fishberg Department of Neuroscience, Department of Genetics and Genomic Sciences and Friedman Brain Institute, Mount Sinai School of Medicine, New York, N.Y., h' Department of Ophthalmology, Tufts University School of Medicine, and 'Sackler School of Graduate Biomedical Sciences, Tufts University School of Medicine, Boston, Mass., USA
\end{abstract}

\section{Key Words}

Allele-specific methylation · Age-related macular degeneration - Copy number variation - Epigenetic analysis - Monozygotic twin pairs .

\footnotetext{
Abstract

Background/Aims: Phenotypic discordance in monozygotic (MZ) twin pairs can have an epigenetic or genetic basis. Although age-related macular degeneration (AMD) has a strong genetic component, few studies have addressed its epigenetic basis. Methods: Using SNP arrays, we evaluated differences in copy number variation (CNV) and allele-specific methylation (ASM) patterns (via methyl-sensitive restriction enzyme digestion of DNA) in MZ twin pairs from the US Twin Study of AMD. Further analyses examined the relationship between ASM and CNVs with AMD by both case/control analysis of ASM at candidate regions and by analysis of ASM
}

\section{KARGER}

E-Mail karger@karger.com

www.karger.com/hhe and CNVs in twins discordant for AMD. Results: The frequency of ASM sites differs between cases and controls in regions surrounding the AMD candidate genes $C F H, C 2$ and CFB. While ASM patterns show a substantial dependence on local sequence polymorphisms, we observed dissimilar patterns of ASM between MZ twins. The genes closest to the sites where discordant MZ twins have dissimilar patterns of ASM are enriched for genes implicated in gliosis, a process associated with neovascular AMD. Similar twin-based analyses revealed no AMD-associated CNVs. Conclusions: Our results provide evidence of epigenetic influences beyond the known genetic susceptibility and implicate inflammatory responses and gliosis in the etiology of AMD.

(c) 2014 S. Karger AG, Basel

J.N. Hutchinson and J. Fagerness contributed equally to this paper. (c) 2014 S. Karger AG, Basel

0001-5652/14/0782-0059\$39.50/0
Andrew Chess and Johanna M. Seddon

Tufts Medical Center

800 Washington St. \#450

Boston, MA 02111 (USA)

E-Mail andrew.chess@mssm.edu or jseddon@tuftsmedicalcenter.org 


\section{Background}

The US Twin Study of Age-Related Macular Degeneration (AMD), a population-based study of monozygotic (MZ) and dizygotic (DZ) twin pairs concordant and discordant for AMD, was established in 1988 to evaluate the relative effects of genetics and the environment on AMD [1]. The results of this study concluded that the heritability of AMD is between 46 and $71 \%$, with more advanced cases having a higher heritability of between 67 and $71 \%$ [1]. As shown in our previous studies of twins, including the study of MZ twin pairs discordant for AMD, environmental factors such as smoking and diet also contribute to this disease, and together with genetic factors they are predictive of the onset and progression of the disease [2-4].

Since genetic susceptibility is a strong determinant of the development and progression of AMD, identical MZ twin pairs, in which one twin is affected with intermediate or advanced disease and the co-twin is free of disease or in the early stages of it, are a unique population to assess somatic differences and how they contribute to AMD. One unexplored source of these differences is post-twinning de novo copy number variation (CNV), and $\mathrm{MZ}$ twins have been reported to display different $\mathrm{CNV}$ profiles [5]. More commonly, differential environmental exposure is thought to cause MZ twin differences, as suggested in our previous report, which showed that smoking and dietary intake of betaine, methionine, and vitamin $\mathrm{D}$ differ between $\mathrm{MZ}$ twins discordant for AMD [3]. It is possible that environmental exposures exert their influence through molecular mechanisms such as DNA methylation [6], an epigenetic process that can influence gene expression [7].

Previous studies of DNA methylation in MZ twins have demonstrated DNA methylation differences between twins [8], particularly as the twins aged [9]. Studies of DNA methylation have traditionally focused on two types of genomic regions: (1) CpG islands which are statistically improbable clusters of CpGs occurring in gene promoters, and (2) differentially methylated regions associated with genomic imprinting, where methylation is associated with one allele in a parent-of-origin manner. Our recent studies $[10,11]$ as well as others $[12,13]$ have demonstrated a novel type of differential methylation where the methylation mark is consistently associated with one allele. Often termed allele-specific methylation (ASM), the phenomenon can be influenced to varying degrees by DNA sequence within a population, ranging from complete association of methylation and genotype (i.e. cis-regulated ASM) [11-13] to more stochastic associations, where either allele may be associated with the methylation mark. As ASM is associated with expression changes in nearby genes, variation in differential methylation among individuals, including MZ twins, has the potential to affect phenotypic variation [12-15].

In this report, we examined whether differences in CNVs and ASM profiles correlated with the AMD phenotype between twins utilizing two sources of data: (1) a standard analysis of genome-wide CNV among 66 individuals (a total of 33 twin pairs: 7 case pairs, 16 control pairs, and 10 phenotypically discordant pairs) using Affymetrix 6.0 SNP arrays, and (2) an assay of ASM, using methyl-sensitive restriction enzyme (MSRE) digestions paired with Affymetrix 6.0 SNP arrays, at almost 100,000 genomic regions in a subset of these samples. The subset for differential methylation analyses included $11 \mathrm{MZ}$ twin pairs (2 with phenotypically discordant AMD status, 4 case pairs and 5 control pairs) and 14 singleton samples.

\section{Methods}

For further details on methods development, see the online supplementary Material (for all online suppl. material, see www. karger.com/doi/10.1159/000362814).

\section{Study Population}

The study population for the US Twin Study of AMD was derived from the National Academy of Sciences - National Research Council World War II Veteran Twin Registry, which includes medical information for 15,924 white male twin pairs born between 1917 and 1927 and was used for both CNV and methylation analyses [16]. Details of the twin study methods have been previously described [1-3]. Briefly, among 12,126 individuals surveyed, 684 (5.6\%) reported having been diagnosed with AMD, and 820 individual twins were enrolled in the Twin Study of AMD, including $381 \mathrm{MZ}$ and DZ pairs and 58 singletons [1]. From this population, we selected $\mathrm{MZ}$ twin pairs discordant for AMD stage. We defined discordant pairs as each co-twin having a different Clinical Age-Related Maculopathy Staging System (CARMS) grade [17] at the last known assessment, with one twin being affected with intermediate AMD (large drusen and/or drusenoid retinal pigment epithelial detachment) or advanced AMD [either geographic atrophy (GA) or neovascular (NV) disease], and the other co-twin with no AMD or in the early stages. Blood samples were drawn, DNA was extracted and cell lines were established using standard procedures. Institutional review boards approved the research protocols and all participants signed consent statements. Research adhered to the tenets of the Declaration of Helsinki.

\section{Macular Characteristics}

Fundus examination and standard fundus photography were performed to record the stage of AMD without knowledge of the zygosity of the twins. Photographs of the macula were graded using a grid according to the Wisconsin Age-Related Macular Degen- 
Table 1. Sample AMD grades

a $\mathrm{CNV}$ analysis of twins

\begin{tabular}{|c|c|c|c|c|c|c|c|}
\hline & \multirow[t]{2}{*}{ Twin-II: } & \multicolumn{3}{|l|}{ Affected } & \multicolumn{3}{|l|}{ Unaffected } \\
\hline & & CARMS 5 & CARMS 4 & CARMS 3 & CARMS 2 & CARMS 1 & total \\
\hline \multicolumn{8}{|l|}{ Twin-I } \\
\hline \multirow[t]{3}{*}{ Affected } & CARMS 5 & 4 & 3 & & 6 & & 13 \\
\hline & CARMS 4 & & & & 3 & 1 & 4 \\
\hline & CARMS 3 & & & & & & 0 \\
\hline \multirow[t]{2}{*}{ Unaffected } & CARMS 2 & & & & & & 0 \\
\hline & CARMS 1 & & & & & 16 & 16 \\
\hline Total & & 4 & 3 & 0 & 9 & 17 & 33 \\
\hline
\end{tabular}

b Methylation analysis of twins

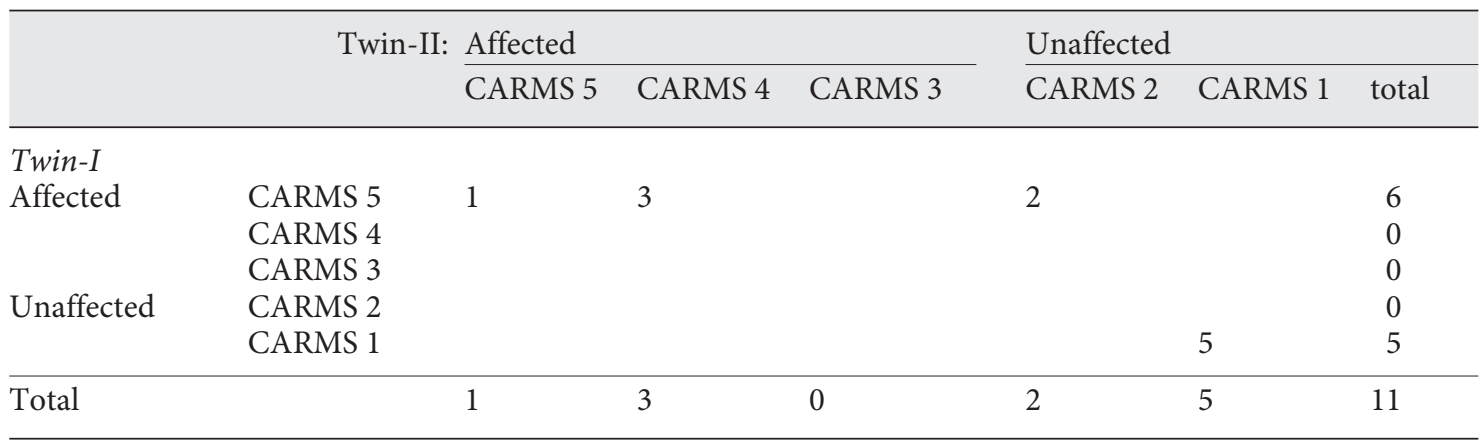

c Methylation analysis of singletons

\begin{tabular}{|c|c|c|c|c|c|c|}
\hline & \multicolumn{3}{|l|}{ Affected } & \multicolumn{3}{|c|}{ Unaffected } \\
\hline & CARMS5 & CARMS4 & CARMS3 & CARMS2 & CARMS1 & total \\
\hline Total & 2 & 1 & & 3 & 8 & 14 \\
\hline
\end{tabular}

Grades of matched twins ( $\mathbf{a}$ and $\mathbf{b}$ ) and singletons (c) used for CNV analyses (a) and methylation analyses (b and c) using CARMS [17].

eration Grading System [18] for a detailed evaluation of macular drusen and retinal pigment characteristics. Study examination data and photographs were also graded by J.M.S. and classified into normal or no AMD (grade 1), early (grade 2), intermediate (grade 3 ), and advanced AMD stages of GA (grade 4) and NV disease (grade 5) according to the CARMS grading system (table 1 and online suppl. table 1) [17].

\section{Zygosity Status and Genotyping}

Each MZ twin pair was genotyped on the Affymetrix 6.0 (Affymetrix Inc., Santa Clara, Calif., USA) platform at the Broad Institute Center for Genotyping and Analysis (Cambridge, Mass., USA). The Affymetrix 6.0 has probe sets against 906,600 SNPs and $940,000 \mathrm{CNV}$ probes. Data for each twin pair were analyzed using PLINK [19] for basic quality assurance and quality control (QAQC) metrics. Samples that performed at acceptable QAQC levels in the SNP-scan part of our GWAS were used for the CNV part of our analysis. Zygosity was determined by an identity-by-descent (IBD) score, which looks at overall genotypic sharing as a function of relatedness. To pass zygosity checks, we required twin pairs to have IBD scores of $>0.95$. Known AMD genes were also genotyped and twins in each pair in the analysis had identical genotypes.

\section{CNV Analyses}

The initial study population consisted of 35 twin pairs. Two of these twin pairs failed the initial genotyping based zygosity checks, so 66 individuals (a total of 33 twin pairs: 7 case pairs, 16 control pairs, and 10 phenotypically discordant pairs) were passed through to the $\mathrm{CNV}$ twin pair analyses. 
CNVs were identified using Birdseye [20], which identifies rare CNVs by integrating intensity data from neighboring probes using a Hidden Markov Model (HMM) on a per-individual basis. Performance is dependent on a number of factors including SNP and copy number probe density, mean intra-individual probe variance and CNV frequency. For each CNV, a logarithm of odds (LOD) score was generated that describes the likelihood of the CNV relative to no CNV over the given interval.

Within the 66 samples, we observed 297,345 unfiltered regions (i.e. without Birdseye baseline filters such as length of deletion event, number of probes identified in the region, contiguity of probes identified in a region) of copy number deletions that differed between twins. We restricted our search to deletions as they have much stronger signal-to-noise signal properties than duplications. A CNV duplication, unless there are multiple duplications, will only provide a 3:2 (one chromosomal duplication) or 4:2 (two chromosomal duplications) ratio, which is much harder to see than a 2:1 (one chromosomal deletion) or 2:0 (two chromosomal deletions) ratio. In order to obtain a high-quality data set, we restricted our analysis to the $307 \mathrm{CNV}$ s with a LOD $>10$ and a physical length $>100 \mathrm{~kb}$ within our analysis set. We imposed an approximately $1 \%$ frequency threshold by removing any $\mathrm{CNV}$ with $>50 \%$ of its length spanning a region with more than 65 out of approximately $6,500 \mathrm{CNV}$ s in the total sample [21]. We also removed events spanning regions of common CNV (>1\%) in the CEU HapMap individuals identified using Affymetrix array data generated in the same laboratory and called with the same analytic pipeline. Additionally, we removed regions identified in the Database of Genomic Variants [22]. As a final step, we joined any CNVs that appeared to be artificially split by the HMM used in analysis, and also removed any CNVs that spanned known large gaps $(>200 \mathrm{~kb})$ in the hg18 reference genome or regions of known rearrangement (e.g.hg18: chr2:88695164-95087413; chr14:104644530106268819; chr22:20797557-21512883) to yield novel CNV deletion events that differ between twins [23].

\section{Methylation Analyses}

Samples

DNA samples for methylation analyses were all derived from whole blood. Cell lines were not considered for these analyses since we found that, in agreement with recent reports [24], methylation patterns differ between lymphoblast cell lines and whole blood DNA derived from the same subjects. Among the 36 samples, 14 were singletons used only for case-control analyses and 22 were twins used in the twin-pair methylation analyses. Of the 14 singletons, 12 samples were derived from twins pairs for which one twin had only cell line DNA and 2 were derived from the two twin pairs which failed the zygosity checks in the CNV QAQC steps described above. The other 22 samples were from 11 twin pairs (table 1 and online suppl. table 1) consisting of 5 concordant control twin pairs (both twins grade 1), 4 concordant case twin pairs and 2 twin pairs discordant for AMD (1 pair with both twins grade 5 and 3 pairs with one twin grade 4 and the other twin grade 5), and 2 twin pairs discordant for AMD (one twin grade 5 and the other twin grade 2). These samples were used in both case-control and twin-pair methylation analyses. To adjust for technical variation, all methylation samples were analyzed as technical replicates on two independent Affymetrix arrays. Probe intensities used in further analyses represent the mean values from these technical replicates.

\section{MSRE Digest and Validation}

For the high-throughput methylation assay, genomic DNA was genotyped on the Affymetrix 6.0 SNP mapping arrays according to the manufacturer's manual (www.affymetrix.com). For methylation analysis, $3 \mu \mathrm{g}$ of genomic DNA was digested at $37^{\circ} \mathrm{C}$ for $16 \mathrm{~h}$ with a MSRE cocktail including Aci I (60 units), BsaH I (3.9 units), Hha I (7.5 units), Hpa II (7.5 units), and HpyCH4 IV (30 units) (New England Biolabs), in a $200-\mu$ l reaction volume with $1 \%$ BSA and 10\% NEB buffer No. 4 (New England Biolabs) and heat inactivated for $20 \mathrm{~min}$ at $60^{\circ} \mathrm{C}$. Samples were ethanol precipitated, washed in $70 \%$ ethanol, and resuspended in reduced EDTA TE ( $5 \mathrm{~mm}$ Tris, $0.1 \mathrm{~mm}$ EDTA) at $50 \mathrm{ng} / \mu \mathrm{l}$. After this pre-treatment, the DNA entered the mapping array procedure; it was further digested with the Nsp I and Sty I restriction enzymes, and fragments of 100-1,100 bp (containing the polymorphic sites to be assessed) were PCR amplified, with the resulting amplicons then end labeled and hybridized to the array. The efficacy of the independent MSRE digestions was determined by examining MSRE action on amplicons with solitary cut sites for a component MSRE in a control mix run alongside the AMD samples. Our results showed that amplicons with single MSRE cut sites, with the exception of HhaI, exhibited greatly reduced combined probe intensities compared to amplicons with no MSRE sites (MNRs) (online suppl. fig. 1). Any amplicons with HhaI sites only (6,132 in total) were ignored in further analyses.

\section{Array Quality Control}

To eliminate replicates with poor array hybridizations, the unnormalized probe intensities of the replicates of MSRE-digested samples were compared by linear regression. All replicate coefficients of determination were within $2.5 \mathrm{SD}$ of the mean of the entire sample set $($ mean $=0.919, \mathrm{SD}=0.125)$. After normalization, the mean coefficient of determination was 0.94 with a SD of 0.028 . All but two samples had coefficients within $2.5 \mathrm{SD}$ of this mean (T39B: 0.86 and T27B: 0.87).

\section{Array Normalization and ASM Detection}

Briefly, invariant probe sets were first quantile-normalized between arrays and the normalized values for the variant probe sets were then interpolated from the normalized invariant probe sets derived from their respective arrays. After various levels of technical cleanup, including eliminating probe sets that performed poorly, ASM at heterozygous SNPs was detected as a sample and SNPspecific normalized deviation from the HapMap-derived heterozygote relative probe intensity (see online suppl. Methods for more details). The potential for artifacts arising from polymorphisms in MSRE recognition sites was eliminated as described previously [11]. Briefly, we excluded all SNPs on the Affymetrix array residing on amplicons containing any known polymorphism in an MSRE recognition site in the dbSNP129 database [25]. These filters were used for all such polymorphisms present at any frequency in the population. Unless otherwise noted, this filter was used in all analyses. Although this filter discards loci that may not be polymorphic for the individuals used in this study, we conservatively chose to ensure robust analyses. For the validation of these methods on known ASM regions, see the online supplementary Results section of the online supplementary Materials. 
Independent Confirmation

We employed 454 large-scale parallel pyrosequencing to examine SNP and CpG site methylation status. Primers for bisulfite PCR (BSP) amplification were designed with Methprimer [26] and BiSearch [27] to uniquely amplify fragments of $<500$ bp encompassing the MSRE sites and target SNP. All amplifications were strand specific and designed to allow observation of the SNP status after bisulfite conversion and amplification; for C/T SNPs, the guanine strand was amplified. Primer sequences (against bisulfiteconverted DNA) and amplicon genomic locations for all amplicons can be found in online supplementary table 2. DNA samples were bisulfite converted with the Qiagen EpiTect 96 Bisulfite Kit according to the manufacturer's instructions, quantitated by Nanodrop and amplified in 96-well plates for 20- $\mu$ l PCR reactions comprised of $10 \mathrm{ng}$ converted DNA, 1 unit Qiagen HotStarTaq Plus, $50 \mathrm{pM}$ of each primer $(2.5 \mu \mathrm{M}$ final concentration $), 1 \times \mathrm{PCR}$ buffer, and $200 \mathrm{nM}$ dNTPs (10 mM final concentration). All amplifications were run for 35 cycles. Product sizes were visually confirmed by agarose gel electrophoresis, quantitated by Picogreen, and equimolar amounts from each target's amplification products mixed for each individual before bar code-indexed 454 pyrosequencing.

After sequencing, pooled reads were deindexed and adapters and low-quality sequences were clipped; reads shorter than 100 were removed. Reads were nondirectionally aligned to amplicon sequences with Bismark [28] with the Bowtie 2 gapped. Bisulfite conversion rates $>95 \%$ were confirmed, and reads not extending over both the amplicon's target SNP and MSRE sites were discarded. Genotypes at target SNPs were called in VCF format with SAMtools pileup [29] with extended BAQ, and a BAQ cutoff of 10 .

All amplicons had a coverage depth of at least 20 reads and genotype qualities of at least 20 in a majority of the samples $(>50 \%)$. SNPs did not significantly deviate $(\mathrm{p}>0.01)$ from the expected Hardy-Weinberg equilibrium within the sample population. Agreement with the expected minor allele frequency, as defined by a relative mean difference $<0.2$ from the expected CEU minor allele frequency (HapMap release 27), was also confirmed for all amplicons. For the results of these validation experiments, see the online supplementary Results section of the online supplementary Materials. All unprocessed data are available by request from Dr. J.M. Seddon.

\section{Results}

\section{CNV Analyses}

After quality control and filtering, we observed a total of $307 \mathrm{CNV}$ s that were not shared between the twins of a twin pair. These 307 unshared CNVs were distributed among the different twin pair types (e.g. case-case, control-control, case-control) rather than concentrated in any twin-pair type. While the majority of these 307 unshared CNVs were commonly observed deletions, 8 were novel CNV deletion events. None of these 8 events were seen in more than one twin pair, and they were also distributed throughout the twin-pair types. These $8 \mathrm{CNV}$ deletion events occur in genomic regions that are mostly devoid of genes, and where genes are present, they are neither implicated in AMD by previous association studies nor are they paralogs of the previously implicated genes.

\section{ASM at Regions Implicated in AMD}

A $\chi^{2}$ analysis of individual MPRs between individuals classified as cases (13 individuals; CARMS grades 3-5) [17] and controls (23 individuals; CARMS 1 and 2) (online suppl. table 1) did not yield any significant results after correction for multiple hypothesis testing (online suppl. fig. 2; Bonferroni adjusted p > 0.05; online suppl. table 3). As ASM changes can correlate across larger genomic regions than assayed by a single MPR [14], we also examined the grouped ASM status of all MPRs in the $1-\mathrm{Mb}$ windows around each of the 12 genetic variants previously implicated in AMD:

(1) rs1061170, or $C F H Y 402 \mathrm{H}$, affecting exon 9 of the $\mathrm{CFH}$ gene on chromosome 1q32, resulting in a substitution of histidine for tyrosine at codon 402 of the $\mathrm{CFH}$ protein [32-34];

(2) rs1410996, an independently associated SNP variant within intron 14 of $\mathrm{CFH}$ [35];

(3) rs10033900, an independently associated SNP located in the linkage peak region of chromosome 4, 2,781 bp downstream of the $3^{\prime}$ untranslated region of CFI [36];

(4) rs9332739, or C2 E318D, a non-synonymous coding SNP variant in exon 7 of $C 2$ resulting in a substitution of aspartic acid for glutamic acid at codon 318 [35, 37];

(5) rs641153, or CFB R32Q, a non-synonymous coding SNP variant in exon 2 of $C F B$ resulting in the substitution of the amino acid glutamine for arginine at codon $32[35,36]$;

(6) rs4711751, a non-coding variant downstream of VEGFA [38],

(7) rs1999930, a non-coding variant near FRK/ COL10A1 [38],

(8) rs10490924, a non-synonymous coding SNP variant in exon 1 of ARMS 2 on chromosome 10 resulting in a substitution of the amino acid serine for alanine at codon $69[39,40]$;

(9) rs10468017, a promoter variant of the Hepatic Lipase C gene LIPC on chromosome 15q22 [21];

(10) rs3764261, a non-coding variant approximately $2.5 \mathrm{~kb}$ upstream of CETP on chromosome 16 [21,38]

(11) rs2230199, or C3 R102G, a non-synonymous coding SNP variant in exon 3 of $C 3$ resulting in the substitution of the amino acid glycine for arginine at codon 102 $[41,42]$, and 
Table 2. Analyses of allele-specific methylation in cases and controls in regions surrounding known AMD variants

a Allele-specific methylation in cases and controls

\begin{tabular}{|c|c|c|c|c|c|c|}
\hline & \multicolumn{2}{|l|}{ Cases } & \multicolumn{2}{|c|}{ Controls } & \multirow{2}{*}{$\begin{array}{l}\mathrm{p} \\
\text { value }\end{array}$} & \multirow{2}{*}{$\begin{array}{l}\text { Percen- } \\
\text { tile }\end{array}$} \\
\hline & MPRs & $\begin{array}{l}\text { ASM } \\
\text { MPRs }\end{array}$ & MPRs & $\begin{array}{l}\text { ASM } \\
\text { MPRs }\end{array}$ & & \\
\hline \multicolumn{7}{|c|}{ 5e5-bp window size } \\
\hline rs 1061170 & 37 & 4 & 59 & 0 & 0.0199 & 2.02 \\
\hline rs1410996 & 37 & 4 & 60 & 0 & 0.0191 & 1.97 \\
\hline rs10033900 & 127 & 5 & 149 & 16 & 0.0404 & 4.07 \\
\hline rs9332739 & 128 & 3 & 158 & 16 & 0.0085 & $0.81^{*}$ \\
\hline rs641153 & 137 & 3 & 166 & 16 & 0.0082 & $0.76^{*}$ \\
\hline rs4711751 & 213 & 17 & 266 & 15 & 0.3586 & 32.15 \\
\hline rs1999930 & 133 & 12 & 140 & 11 & 0.8286 & 75.00 \\
\hline rs10490924 & 188 & 14 & 263 & 25 & 0.4994 & 45.23 \\
\hline rs10468017 & 391 & 27 & 496 & 32 & 0.7879 & 71.50 \\
\hline rs3764261 & 181 & 18 & 274 & 26 & 0.8726 & 80.09 \\
\hline rs2230199 & 151 & 7 & 213 & 10 & 1.0000 & 99.93 \\
\hline rs9621532 & 274 & 19 & 379 & 40 & 0.1285 & 12.12 \\
\hline \multicolumn{7}{|c|}{ 1e6-bp window size } \\
\hline rs 1061170 & 84 & 11 & 145 & 2 & 0.0004 & $0.06^{* *}$ \\
\hline rs1410996 & 84 & 9 & 144 & 2 & 0.0025 & $0.28^{* *}$ \\
\hline rs10033900 & 350 & 28 & 430 & 39 & 0.6103 & 57.83 \\
\hline rs9332739 & 346 & 16 & 425 & 35 & 0.0574 & 5.95 \\
\hline rs641153 & 350 & 16 & 439 & 36 & 0.0438 & 4.58 \\
\hline rs4711751 & 331 & 25 & 431 & 20 & 0.2052 & 20.24 \\
\hline rs1999930 & 333 & 31 & 460 & 33 & 0.2924 & 28.83 \\
\hline rs10490924 & 382 & 45 & 567 & 64 & 0.8359 & 79.34 \\
\hline rs10468017 & 561 & 35 & 664 & 41 & 1.0000 & 99.98 \\
\hline rs3764261 & 389 & 30 & 511 & 37 & 0.7989 & 75.64 \\
\hline rs2230199 & 302 & 9 & 434 & 19 & 0.4340 & 42.06 \\
\hline rs9621532 & 477 & 49 & 644 & 66 & 1.0000 & 99.98 \\
\hline
\end{tabular}

For the two window sizes shown, $500 \mathrm{~kb}$ and $1 \mathrm{Mb}$ centered on the AMD-associated variant, ASM counts were summed for all MPRs within the window in both cases and controls and Fisher's exact tests performed. These values were compared to values derived from 10,000 identically sized, randomly located windows drawn from the same data. One region surrounding two variants (rs1061170 and rs1410996 located approx. $38 \mathrm{~kb}$ apart on chromosome 1) showed more differences between cases and controls than $99 \%$ of randomly drawn windows of $1 \mathrm{Mb}$ in size and more than $95 \%$ of randomly drawn windows of $500 \mathrm{~kb}$ in size. Another region surrounding two variants (rs9332739 and rs6411536 located approx. $10 \mathrm{~kb}$ apart on chromosome 6) showed more significant differences between cases and controls than more than $99 \%$ of randomly drawn windows of $500 \mathrm{~kb}$ in size and almost $95 \%$ of randomly drawn windows of $1 \mathrm{Mb}$ in size.

* Significant at $\mathrm{p}<0.1$ after multiple testing adjustment. ${ }^{* *}$ Significant at $\mathrm{p}<0.05$ after multiple testing adjustment. b Known AMD variants

\begin{tabular}{llll}
\hline SNP & Proximal gene & Chromosome & Position \\
\hline rs1061170 & CFH & 1 & 194925860 \\
rs1410996 & CFH & 1 & 194963556 \\
rs10033900 & CFI & 4 & 110878516 \\
rs9332739 & C2 & 6 & 32011783 \\
rs641153 & CFB & 6 & 32022159 \\
rs4711751 & VEGFA & 6 & 43936560 \\
rs1999930 & FRK/COL10A1 & 6 & 116493827 \\
rs10490924 & ARMS2/HTRA1 & 10 & 124204438 \\
rs10468017 & LIPC & 15 & 56465804 \\
rs3764261 & CETP & 16 & 55550825 \\
rs2230199 & C3 & 19 & 6669387 \\
rs9621532 & TIMP3 & 22 & 31414511 \\
\hline
\end{tabular}

(12) rs9621532, a variant upstream of TIMP3 [21, 43].

While the majority of windows $(8 / 12)$ showed no significant association of ASM occurrence with either case or control status, 2 largely overlapping windows centered on the CFH locus (rs1061170 and rs1410996) had significant differences (Bonferroni adjusted $\mathrm{p}$ values $<0.05$ at 0.0072 and 0.0336 , respectively). Two overlapping windows centered on SNPs within the C2 and CFB loci (rs9332739 and rs641153) also showed significant differences (Bonferroni adjusted $\mathrm{p}$ values $<0.1$ at 0.0972 and 0.0912, respectively) in ASM levels between cases and controls. To account for any correlation structure within our samples which might confound these results, we compared them to results from identically structured tests of 10,000 randomly drawn windows of equal base pair size and equal or higher MPR counts; the AMD SNPcontaining windows represented genomic regions with some of the highest differences in ASM levels between cases and controls (table 2, fig. 1). Taken together, these results and the large overlap of the two windows within each genomic region indicate the presence of 2 genomic regions with differential levels of ASM in patients with a differential AMD status.

\section{MZ Twins Exhibit Different ASM Patterns}

We examined the overall level of disagreement between twins for ASM and observed 2 types of methylation scenarios: (1) allelic differences, where both twins showed ASM at an MPR, but for different alleles, and (2) ASM occurrence differences, where one twin exhibits ASM at an MPR and the other twin exhibits biallelic methylation (fig. 2). We observed very few cases of the first scenario;
Hutchinson et al. 
Table 3. Analyses of allele-specific methylation allele preference agreement between twins

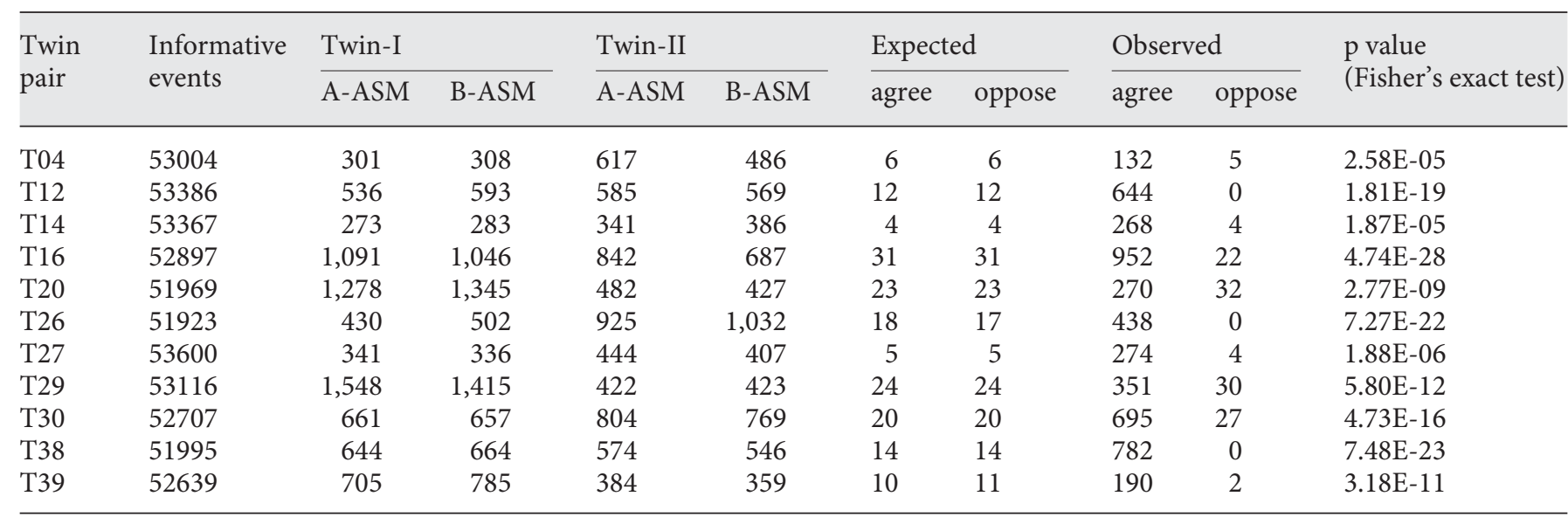

Results are shown for ASM allele preferences for informative MPRs (heterozygous genotype, above intensity cutoff) in Twin-I and Twin-II of a twin pair; the number of ASM events associated with either allele A (A-ASM) or allele B (B-ASM) for each twin are shown (the remaining informative MPRs show biallelic methyla- tion in at least one twin). The observed numbers of matching (agree) or opposite (oppose) allele preference between twins are shown as compared to the null hypothesis of random preference by Fisher's Exact tests.

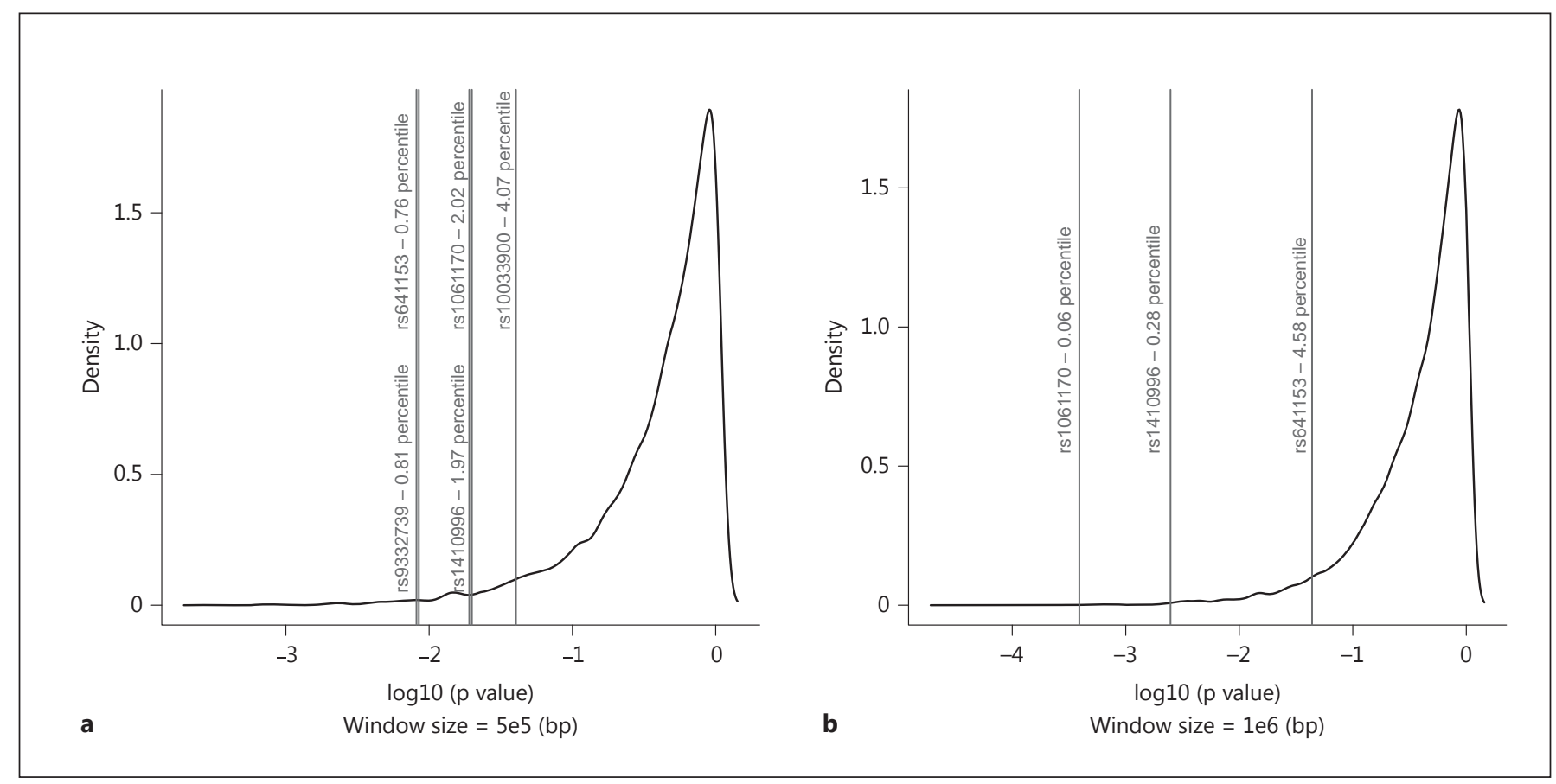

Fig. 1. ASM differences between cases and controls in the regions surrounding the genetic variants with known AMD associations. Distributions of $p$ values from Fisher's exact tests of ASM counts in cases and controls are derived from randomly drawn MPR-containing windows of $500 \mathrm{~kb}(\mathbf{a})$ and $1 \mathrm{Mb}(\mathbf{b})$. Vertical lines indicate the positions of $\mathrm{p}$ values derived from the windows surrounding the known AMD variants found to be within the 5th percentile of the distribution. The respective variant identities and percentiles are indicated to the left of the lines. 


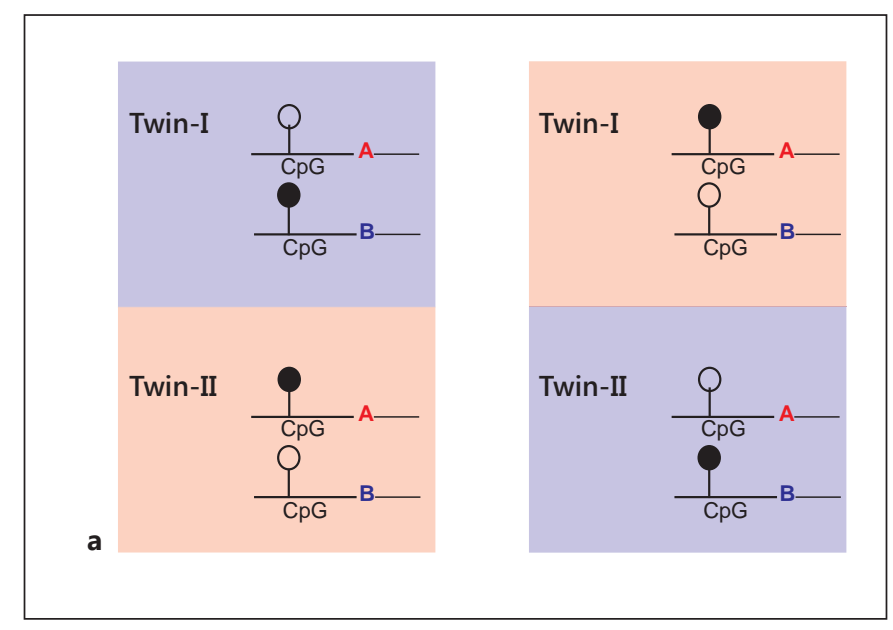

Fig. 2. Differential ASM scenarios in MZ twins. Potential modes of differing ASM calls between MZ twins are shown. In the first model (a), the identity of the allele associated with methylation distinguishes the twins: Twin-I or Twin-II exhibits methylation associated with the A allele (upper row) and the other twin exhibits methylation associated with the B allele (lower row). Alternatively, in a second model (b), the differential occurrence of ASM distinguishes the twins: Twin-I or Twin-II shows ASM (associated with either one of the A or B alleles), and the other twin exhibits biallelic methylation.

less than one would expect were allele preference random (table 3). This supports our prior work showing the prevalence of SNP-influenced ASM across the genome [11]. The second scenario is more prevalent; while most ASM events are present in both twins, we did observe instances where, for up to $5.2 \%$ of MPRs, one twin exhibits ASM and the other does not. To account for the possibility of these instances resulting from technical variation, we leveraged the two technical replicate arrays, comparing the level of disagreement found between all replicates between twins (4 comparisons) to that found when comparing replicates from the same twin ( 2 comparisons). We observed that for the majority of whole blood samples examined, biological ASM differences exceed technical noise, i.e. the percentage of ASM disagreements between twins at these MPRs (median 2.5\% of MPRs) exceeds the percentage of ASM disagreements observed when comparing replicates from an individual twin (median 2.05\% of MPRs) (fig. 3). Thus, while most of the disagreements are due to technical noise, some of the differences reflect stable differences between the twins or, alternatively,

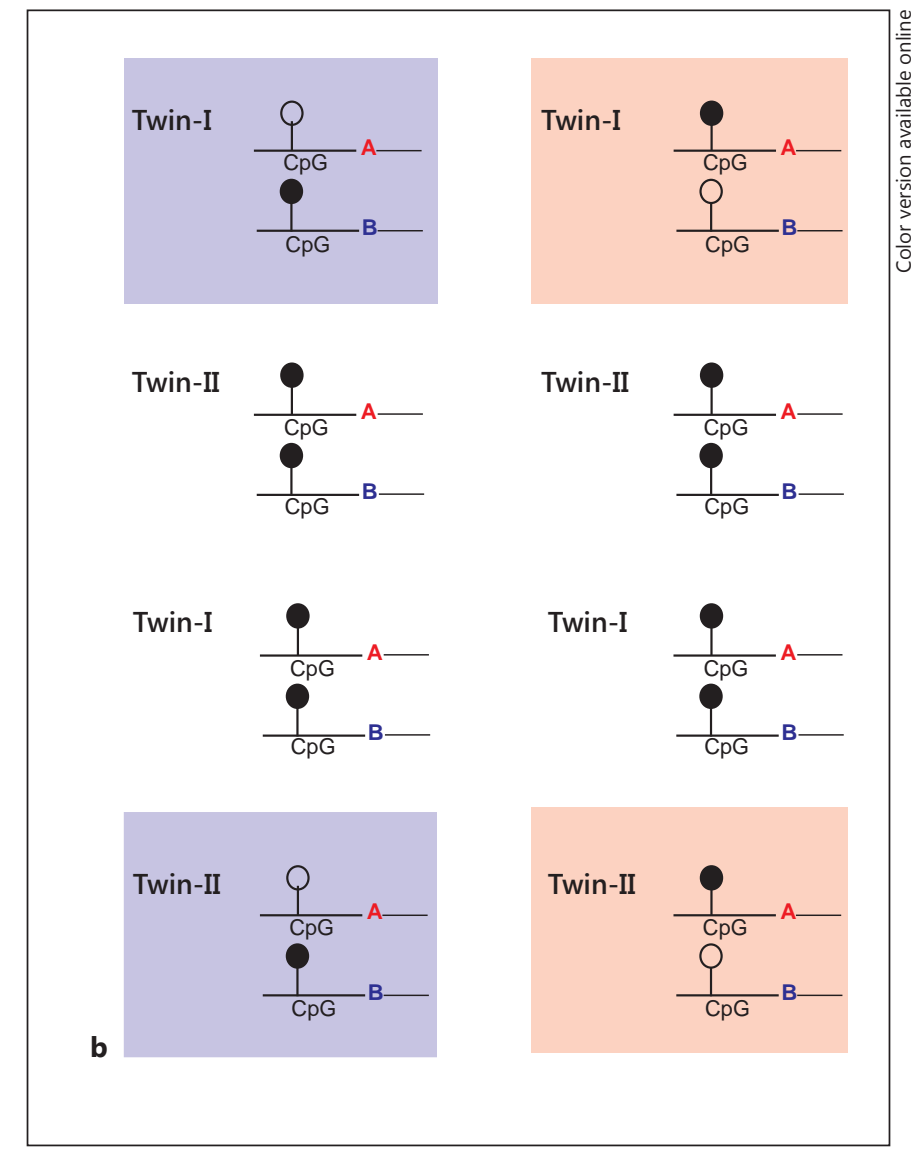

could reflect differences we would have observed if we had compared samples taken at different time points from the same individual.

ASM Differs in Twins with Discordant AMD Status at Regions Proximal to Genes Implicated in Gliosis

We extended these results to search for genomic regions with consistent ASM differences between the discordant twins. For these twins, we did not observe any instances where both twins showed ASM at an MPR, but for different alleles (fig. 2a; e.g. regions where, for both discordant twin pairs, the cases exhibited methylation associated with the A allele and the controls exhibited methylation associated with the B allele). Instead, we observed the differential occurrence of ASM between the discordant twins (fig. 2b), where at 42 MPRs, for both discordant twin pairs, one twin showed biallelic methylation and the other had ASM (e.g. regions where, for both discordant twin pairs, the cases showed either ASM-A or ASM-B and the controls exhibited biallelic methylation) (table 4). To determine how likely we were to see this 


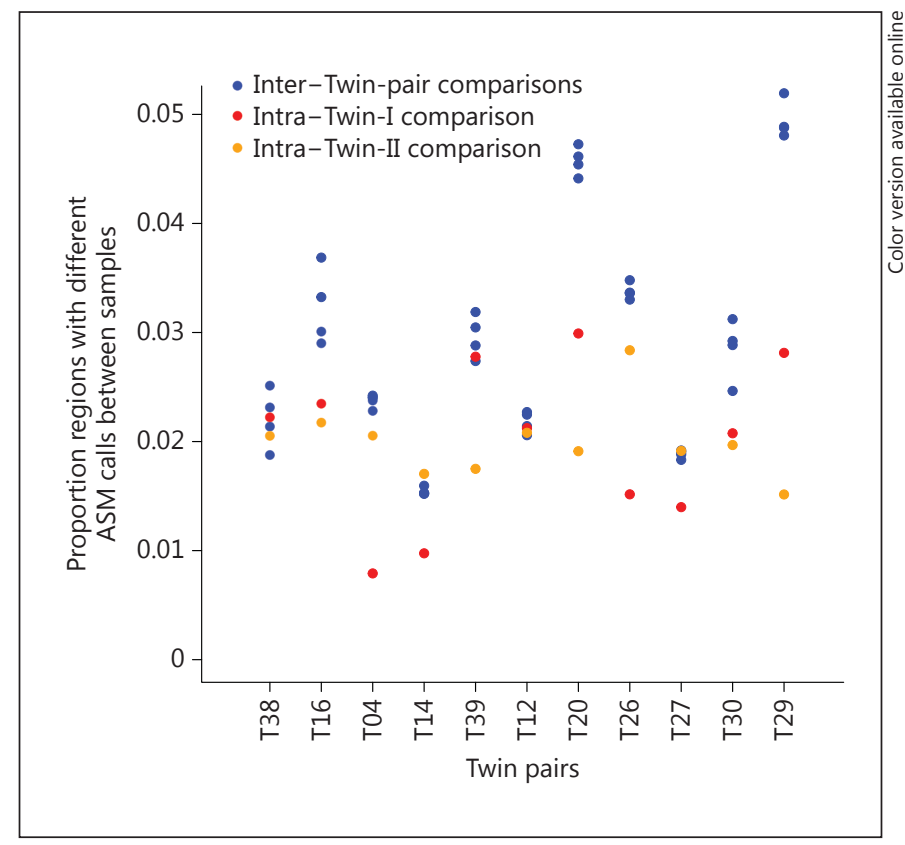

Fig. 3. ASM patterns between MZ twins. Each circle represents the percentage of regions found to disagree for ASM between the two sample comparisons. These results are derived from either technical replicates drawn from an individual twin or comparisons between the replicates from each of the different twins. Results are shown for an approach in which ASM calls were more extreme than a Z-score cutoff excluding $99 \%$ of MNRs (1\% false positive rate) and non-ASM calls less extreme than $95 \%$ of MNRs (i.e. included within $95 \%$ of the MNR distribution). In this approach, SNPs with Z-score values more extreme than the $99 \% \mathrm{Z}$-score cutoff in one twin (i.e. ASM) and between 95 and $99 \%$ in the other twin (i.e. neither ASM nor non-ASM) were excluded as indeterminate. Biological differences between twins that cannot be explained by technical noise are consistent with the inter-twin-pair comparisons having greater values than both intra-twin-pair comparisons. The first five twin pairs are concordant controls, the next four are concordant cases and the final two twin pairs are discordant twins.

number of discordant ASM MPRs at random, we performed a permutation test of the data set, shuffling the discordant and concordant assignments of the twin pairs; 2,217 of the 10,000 of the permuted data sets resulted in at least the same number of methylation differences between the discordant twin pairs to yield a $\mathrm{p}$ value of 0.2217 . To determine if any biological functions might be associated with these particular MPRs, we performed a gene ontology analysis of the genes closest to these 42 MPRs (as determined by the Affymetrix SNP 6.0 hg19 annotations). Our results showed this list of genes to be enriched for genes involved in the process of gliogenesis.
Specifically, 36 of the 42 genes had a gene ontology annotation, and 4 of these 36 genes were contained within the gliogenesis annotation, yielding an adjusted $p$ value of 0.0045 (via the g:SCS algorithm, a multiple-test corrected $\mathrm{p}$ value which accounts for the hierarchical structure within gene ontology terms [44]).

\section{Discussion}

This work represents one of the first genome-wide analyses of differential ASM and DNA methylation within several MZ twin pairs participating in an AMD study. This work also represents the first evaluation of CNVs in $\mathrm{MZ}$ twin pairs with concordant and discordant AMD phenotypes. In addition to identifying smaller (approx. $0.1-1.1 \mathrm{~kb}$ ) differentially methylated genomic regions in MZ twins discordant for AMD, we also demonstrate that larger genomic regions $(0.5-1 \mathrm{Mb})$ containing known AMD-associated variants are differentially methylated according to the AMD status. Our results extend our previous AMD studies with this twin cohort and, while there is no incontrovertible evidence of an epigenetic signature for AMD, our results do suggest candidate epigenetic targets underlying the discordant phenotypes observed in twins [3].

There are few previous studies examining CNV differences between MZ twins. While Bruder et al. [5] claimed they could see many somatic differences between subjects, we took a more conservative approach to setting thresholds for calling these differential CNV events. Upon deeper examination of the underlying data, we found that frequently, when one twin reached the calling threshold, the other twin showed sub-threshold levels of signal. These results indicate that the differences in CNV deletions we do observe between twins are likely driven by small differences in signal between twins, and may not represent true $\mathrm{CNV}$ differences. In support of our conservative approach and the resultant low number of $\mathrm{MZ}$ twin- pair CNV differences, somatic mutations have historically been estimated to occur once every $100 \mathrm{Mb}$, yielding only about 30 events per individual across the genome. While it is difficult to determine when these events occur, we would expect any of the mutations occurring very early during embryogenesis prior to the 'twinning' event to be shared between MZ twins. Therefore, the expectation, which is fulfilled by our results, is for relatively few of these events to be seen between $\mathrm{MZ}$ twins. 
Table 4. SNPs discordant for ASM incidence in monozygotic twins discordant for AMD

\begin{tabular}{|c|c|c|c|c|c|c|c|c|}
\hline \multirow[t]{2}{*}{ ProbeID } & \multicolumn{2}{|c|}{$\begin{array}{l}\text { Discordant twin } \\
\text { status }\end{array}$} & \multicolumn{3}{|l|}{ SNP } & \multicolumn{3}{|l|}{ Closest gene } \\
\hline & T30 & T29 & ID & Chr & position & $\begin{array}{l}\text { relation } \\
\text { to gene }\end{array}$ & $\begin{array}{l}\text { distance from } \\
\text { TSS (if not genic) }\end{array}$ & symbol \\
\hline SNP_A-2083845 & BI-B & BI-B & rs13411523 & 2 & 114454164 & downstream & 21527 & ACTR3 \\
\hline SNP_A-2021856 & A-BI & A-BI & rs11679258 & 2 & 241441734 & upstream & 15100 & AGXT \\
\hline SNP_A-8484764 & BI-B & BI-B & rs6140071 & 20 & 6654493 & upstream & 42251 & BMP2 \\
\hline SNP_A-2210596 & B-BI & B-BI & rs7909808 & 10 & 78062324 & downstream & 75194 & C10orf11 \\
\hline SNP_A-8559771 & BI-A & BI-A & rs9384701 & 6 & 109664235 & downstream & 72429 & C6orf182 \\
\hline SNP_A-8717023 & A-BI & A-BI & rs8063808 & 16 & 47795251 & downstream & 74460 & CBLN1 \\
\hline SNP_A-2133676 & A-BI & A-BI & rs10470437 & 3 & 133265423 & upstream & 28889 & CPNE4 \\
\hline SNP_A-8573108 & $\mathrm{B}-\mathrm{BI}$ & $\mathrm{B}-\mathrm{BI}$ & rs2693022 & 2 & 15842155 & downstream & 153479 & DDX1 \\
\hline SNP_A-4282113 & A-BI & A-BI & rs9309883 & 3 & 82097118 & upstream & 203478 & GBE1 \\
\hline SNP_A-8392053 & A-BI & A-BI & rs2017257 & 17 & 40352675 & upstream & 4235 & GFAP \\
\hline SNP_A-4284358 & B-BI & B-BI & rs12478085 & 2 & 206766767 & intron & & GPR1 \\
\hline SNP_A-8321092 & B-BI & B-BI & rs8076416 & 17 & 74822048 & intron & & hCG_1776007 \\
\hline SNP_A-1867638 & A-BI & A-BI & rs2461056 & 8 & 80836175 & downstream & 2625 & HEY1 \\
\hline SNP_A-8425957 & A-BI & A-BI & rs11207325 & 1 & 59136207 & upstream & 113834 & JUN \\
\hline SNP_A-2246766 & BI-B & BI-B & rs6985117 & 8 & 111591041 & upstream & 534906 & KCNV1 \\
\hline SNP_A-8529083 & A-BI & A-BI & rs10446676 & 4 & 36562606 & upstream & 360478 & KIAA1239 \\
\hline SNP_A-4196589 & B-BI & B-BI & rs10457178 & 6 & 108948401 & intron & & LACE1 \\
\hline SNP_A-8564552 & A-BI & A-BI & rs130993 & 22 & 32897763 & upstream & 251347 & LARGE \\
\hline SNP_A-1855257 & B-BI & B-BI & rs962885 & 17 & 41291420 & intron & & LOC100128977 \\
\hline SNP_A-4298178 & B-BI & B-BI & rs1200470 & 13 & 31010348 & upstream & 201330 & RXFP2 \\
\hline SNP_A-8634510 & A-BI & A-BI & rs 941597 & 14 & 93883114 & upstream & 23673 & SERPINA6 \\
\hline SNP_A-8698392 & A-BI & A-BI & rs7824948 & 8 & 14987154 & intron & & SGCZ \\
\hline SNP_A-8607148 & A-BI & A-BI & rs1731847 & 7 & 155348283 & upstream & 50555 & $\mathrm{SHH}$ \\
\hline SNP_A-8434018 & A-BI & A-BI & rs13179872 & 5 & 128177775 & upstream & 151333 & SLC27A6 \\
\hline SNP_A-4280941 & BI-B & BI-B & rs 454693 & 5 & 87579969 & intron & & TMEM161B \\
\hline SNP_A-2065284 & A-BI & A-BI & rs955336 & 1 & 100881570 & upstream & 76314 & VCAM1 \\
\hline SNP_A-1788455 & A-BI & $\mathrm{A}-\mathrm{BI}$ & rs16963005 & 19 & 34756795 & downstream & 9729 & VSTM2B \\
\hline SNP_A-1953456 & A-BI & A-BI & rs4796421 & 17 & 7320374 & intron & & ZBTB4 \\
\hline
\end{tabular}

Results are shown for an approach in which ASM calls were based on Z-score cutoffs excluding $99 \%$ of MNRs (1\% false positive rate) and any non-ASM calls less extreme than $95 \%$ of MNRs (i.e. included within $95 \%$ of the MNR distribution). In this approach, regions with Z-score values more extreme than the Z-score cutoff in one twin (i.e. ASM) and between 95 and 99\% in the other twin (i.e. neither ASM nor non-ASM) were excluded as indeterminate. The 42 MPRs shown exhibit consistent ASM incidence discordance in twin pairs discordant for AMD, where the ASM call for the twins within the twin pair are separated by a dash. $\mathrm{BI}=$ biallelic methylation; $\mathrm{A}=\mathrm{ASM}$ of the A allele; $\mathrm{B}=\mathrm{ASM}$ of the $\mathrm{B}$ allele; $\mathrm{BI}-\mathrm{BI}=$ biallelic methylation in both twins; A-A = ASM of the A allele in both twins; B-B = ASM of the $\mathrm{B}$ allele in both twins. The code before the dash indicates the ASM status of the unaffected twin (control), and the code after the dash that of the affected twin (case). Affymetrix annotations for genomic location and relationship to the closest gene are also shown for each of these variants. 
Previous attempts to associate methylation changes with phenotypic traits in discordant twins have demonstrated differences between MZ twins within a pair but have largely been limited in genomic scope. Multiple studies targeting $\mathrm{CpG}$ islands have shown suggestive results; Javierre et al. [45] observed widespread changes in the DNA methylation status of a significant number of CpGcontaining promoters of genes associated with immune function in $5 \mathrm{MZ}$ twin pairs discordant for systemic lupus erythematosus. Using the Illumina $27 \mathrm{~K}$ platform (which largely targets $\mathrm{CpG}$ islands of cancer-associated genes), Dempster et al. [46] found a significant enrichment of epigenetic changes in biological pathways relevant to psychiatric disorder and neurodevelopment in 22 twin pairs discordant for major psychosis, while Wang et al. [47] observed methylation differences in 2 genes between 7 twin pairs discordant for obesity. In contrast, a study of $3 \mathrm{MZ}$ twin pairs discordant for multiple sclerosis examined genome-wide DNA methylation using next-generation bisulfite sequencing and did not find any methylation changes associated with the phenotype [48]. Far fewer studies have examined the association of ASM changes with phenotypic traits and none have done so genome wide. Stepanow et al. [49] showed ASM of MCHR1 to be associated with body mass index. In cancer progression, Kang et al. [50] reported an association of p14ARF (CDKN2A) polymorphisms with the likelihood of methylation of this gene within colorectal cancers, and Boumber et al. [51] found an indel polymorphism in PDLIM4 which influences the methylation of this gene in leukemia and colon cancer. Wei et al. [52] examined methylation patterns in 3 twin pairs ( $1 \mathrm{MZ}$ and $2 \mathrm{DZ}$ ) discordant for $A M D$, assaying methylation levels of promoter region genes in PBMCs using MeDip-Chip and NimbleGen $\mathrm{Hu}$ man DNA Methylation 2.1M Deluxe Promoter Arrays. In contrast to our results, they reported consistent differential methylation patterns upstream of IL17RC in these 3 twin pairs. Though other groups have failed to replicate these results [53], the differences between our results and those reported by Wei et al. are due to differing definitions of differential methylation. While Wei et al. define differential methylation as a binary difference in overall methylation between discordant twins (i.e. $0 \%$ methylation in one twin vs. $>0 \%$ methylation in the other), we searched for changes in ASM patterns which present themselves as changes from $100 \%$ methylation (biallelic methylation) to $50 \%$ methylation (ASM). As such, Wei et al. would not have detected our loci. Furthermore, as we screened out loci with overall low intensity levels, we did not examine the unmethylated loci described by Wei et al. [52].

(Epi)Genetic Analyses of MZ Twins Discordant for AMD
Given our focus on rare deletion events, it is perhaps not surprising that we found only $8 \mathrm{CNV}$ differences between MZ twin pairs. Even though we were conservative in calling differential CNV events, we did find several instances of unique $\mathrm{CNV}$ events in one twin of a pair. However, due to the rarity of these events and the relatively large amount of the variance already explained for this disease, we found no statistically significant result showing any of these CNVs to be associated with AMD.

In contrast, our analysis of differential methylation revealed some methylation events associated with AMD. In particular, analyses of large genomic regions from $0.5-1$ $\mathrm{Mb}$ in size near complement factor genes uncovered evidence of differential ASM in these regions between cases and controls. We also found smaller genomic regions with ASM differences between MZ twins discordant for AMD near genes involved in gliogenesis. Taken together, these results further implicate the role of inflammation and glial cell proliferation in AMD progression.

A recent study by Hunter et al. [54] used the Illumina $27 \mathrm{~K}$ platform to assess methylation in a case-control study of postmortem retina pigment epithelium/choroid samples. In contrast to the results we report here, Hunter et al. did not report methylation changes upstream of either $\mathrm{CFH}$ or CFB. However, we report results for large regions (0.5-1 Mb) composed of multiple MPRs that only partially overlap CpGs assayed by both studies. As such, it is possible that (a) the signal we observe is driven by $\mathrm{CpGs}$ not located within the targets assayed by Hunter et al., or that (b) our signal represents an aggregate of signals from multiple amplicons that would not be detected as individual results from CpGs. This latter hypothesis is supported by our failure to find significant differences in ASM in individual MPRs (online suppl. table 3).

It is possible that the methylation changes we observe in whole blood directly influence AMD progression through this cell population's known effects on both the inflammatory and complement responses. Given previous observations of the lack of tissue specificity of ASM [11], it is possible that these methylation differences reflect a broader methylation status within multiple tissues that only manifests itself in combination with other factors within a particular susceptible cell type such as the retinal pigment epithelium. However, given the demonstrated existence of tissue-specific ASM [13, 55], it is possible that the observed methylation changes are the result of differences in the proportions of cell types in the samples examined. While this is unlikely the case in our casecontrol analyses, which involve multiple independent samples, the differences observed between discordant 
twins could be the result of different cell type proportions within the twin blood samples. Lacking prior data on tissue representation, we resorted to examining whether any of the loci we identified in twin pairs with discordant ASM also showed tissue specific methylation. None of the 100 tissue-specific loci identified by Houseman et al. [56] or of the 679 tissue-specific loci identified by Glossop et al. [57] are located within the amplicons identified in our discordant twin study. Of course, this does not eliminate the possibility that these discordant MPRs are as yet unidentified tissue-specific loci, and our results should be interpreted with this in mind.

One smaller region of interest that was identified by our analysis of the discordant MZ twins was that of the MPR-encompassing rs2017257, located downstream of kinesin family member $18 \mathrm{~B}$ and approximately $4.2 \mathrm{~kb}$ upstream of the glial fibrillary acidic protein gene (GFAP). GFAP is an intermediate filament protein expressed in numerous cell types of the central nervous system and is a primary marker of astrocytes [58]. Gliosis, an inflammatory injury response involving astrocyte proliferation and glial scarring during wound healing, is associated with increased levels of GFAP expression [59]. Importantly, both gliosis and increased GFAP levels have been reported to be associated with AMD [58, 60-62]. In this context, the discordant ASM signal observed in the region encompassing rs11949422 is also interesting. This region overlaps some isoforms of the gene for cAMP-specific phosphodiesterase 4D (PDE4D). Rolipram, an inhibitor of phosphodiesterase 4, can attenuate gliosis [63] indicating a potential role for this gene in gliosis and AMD progression. While the exact molecular effect of the differential methylation event near GFAP awaits further research, ENCODE data do identify the MPR's genomic coordinates as a strong CTCF-based insulator in multiple cell types [64]; DNA methylation of a CTCF-binding site can disrupt its insulator function [65]. It is possible that the biallelic methylation observed at this MPR in discordant twin cases results in lower levels of CTCF binding as compared to that of the discordant twin controls which have ASM at this MPR. This in turn could result in a decreased CTCF insulator function and an increased expression of GFAP within the discordant case twins.

Findings of a genetic influence on differential methylation raise the possibility that ASM differences observed between cases and controls at larger genomic regions near known AMD variants originate from these variants themselves. In this respect, we note that the rs1410996 variant near $\mathrm{CFH}$ is noncoding [35], suggesting its potential regulatory role in AMD progression. The risk allele frequencies of rs1410996 in cases (73.1\%) and controls $(50 \%)$ vary significantly $(p<0.05)$, raising the possibility of genetic influence on the observed ASM differences. In contrast, the risk allele frequencies for AMD variants near $C 2$ and $C F B$ (rs9332739 and rs641153) are essentially equal between cases $(92.3 \%$ and $100 \%$, respectively) and controls (100\% for both), suggesting a nongenetic origin for the observed ASM differences between cases and controls.

While the ASM differences observed in genetically identical MZ twins discordant for AMD do not appear to be the result of genetic differences, it is difficult to differentiate between the observed ASM changes as either a cause or effect of AMD (much like with expression changes). In this respect, as noted above, detection of these methylation differences in whole blood derived DNA could be interpreted as an organism-wide epigenetic response to an external stimulus that manifests within a susceptible cell type. Attempting to examine this possibility, we investigated whether the presence of external stimuli in our patients - such as smoking history and body mass index (BMI), known risk factors for AMD [2-4] - correlated with twin discordance and case/control differences in outcome and ASM patterns/levels. We were, however, unable to detect any statistically significant patterns or changes in ASM that correlated with these external events within our small sample set (data not shown).

This study has the advantages of a well-characterized population examined according to the rigorous standard protocols with longitudinal follow-up to obtain the most recent AMD grade. Although we took a very conservative approach towards CNV detection and calling, we did see unique events in one MZ twin and it remains possible that these early somatic mutations could give rise to some of the phenotypic discordance observed in MZ twins. Although our results do not explain AMD discordance between MZ twins, we have helped to develop a foundation on which the field can build its knowledge of somatic structural variation in genetically identical subjects. Further improvements upon previous studies include its genome-wide unbiased examination of ASM through the use of a platform specifically designed for differential allele detection. We observed many suggestive results, particularly those involving genomic regions proximal to loci involved in the inflammatory and wound-healing responses. Although this is the largest twin study of AMD done for these types of analyses, the sample size was small and some associations may have been missed. 


\section{Conclusions}

In summary, we examined the role of CNV and differential methylation in AMD in a genetically constrained cohort of MZ twins and identified differentially methylated regions in discordant twins with biologically suggestive roles. Our results provide no definite evidence that epigenetics plays a central role in AMD, but they do provide some evidence of epigenetic influences beyond the known genetic susceptibility and further implicate inflammatory responses and gliosis in the etiology of AMD.

Larger studies of carefully selected twins or singletons targeted to these genomic regions may be an effective way of further assessing the effect of epigenetic modifications at these locations in AMD. Given the malleable nature of epigenetic changes, these results may eventually provide therapeutic targets for this important and increasingly prevalent disease.

\section{Acknowledgements}

We thank Yi Yu and Lillian Merriam for their constructive criticism of the manuscript. This work was supported in part by grants R01-EY11309 from the National Institutes of Health, Bethesda, Md.; Massachusetts Lions Eye Research Fund, Inc.; unrestricted grant from Research to Prevent Blindness, Inc., New York, N.Y.; the American Macular Degeneration Foundation, Northampton, Mass.; donations to the Macular Degeneration Research Fund of the Ophthalmic Epidemiology and Genetics Service, New England Eye Center, Tufts Medical Center, Tufts University School of Medicine, Boston, Mass.

\section{References}

1 Seddon JM, Cote J, Page WF, Aggen SH, Neale MC: The US twin study of age-related macular degeneration: relative roles of genetic and environmental influences. Arch Ophthalmol 2005; 123:321-327.

-2 Seddon JM, George S, Rosner B: Cigarette smoking, fish consumption, omega-3 fatty acid intake, and associations with age-related macular degeneration: the US Twin Study of Age-Related Macular Degeneration. Arch Ophthalmol 2006;124:995-1001.

- 3 Seddon JM, Reynolds R, Shah HR, Rosner B: Smoking, dietary betaine, methionine, and vitamin D in monozygotic twins with discordant macular degeneration: epigenetic implications. Ophthalmology 2011;118:13861394.

-4 Seddon JM, Reynolds R, Yu Y, Daly MJ, Rosner B: Risk models for progression to advanced age-related macular degeneration using demographic, environmental, genetic, and ocular factors. Ophthalmology 2011;118: 2203-2211.

-5 Bruder CEG, Piotrowski A, Gijsbers AACJ, Andersson R, Erickson S, Diaz de Ståhl T, et al: Phenotypically concordant and discordant monozygotic twins display different DNA copy-number-variation profiles. Am J Hum Genet 2008;82:763-771.

-6 Dolinoy DC, Weidman JR, Jirtle RL: Epigenetic gene regulation: linking early developmental environment to adult disease. Reprod Toxicol 2007;23:297-307.

7 Jaenisch R, Bird A: Epigenetic regulation of gene expression: how the genome integrates intrinsic and environmental signals. Nat Genet 2003;33(suppl):245-254.

8 Fraga MF, Ballestar E, Paz MF, Ropero S, Setien F, Ballestar ML, et al: Epigenetic differences arise during the lifetime of monozygotic twins. Proc Natl Acad Sci USA 2005;102: 10604-10609.
9 Boks MP, Derks EM, Weisenberger DJ, Strengman E, Janson E, Sommer IE, Kahn RS, Ophoff RA: The relationship of DNA methylation with age, gender and genotype in twins and healthy controls. PLoS One 2009; 4:e6767.

10 Hellman A, Chess A: Gene body-specific methylation on the active $\mathrm{X}$ chromosome. Science 2007;315:1141-1143.

11 Hellman A, Chess A: Extensive sequence-influenced DNA methylation polymorphism in the human genome. Epigenetics Chromatin 2010;3:11.

12 Kerkel K, Spadola A, Yuan E, Kosek J, Jiang L, Hod E, et al: Genomic surveys by methylation-sensitive SNP analysis identify sequencedependent allele-specific DNA methylation. Nat Genet 2008;40:904-908.

13 Schalkwyk LC, Meaburn EL, Smith R, Dempster EL, Jeffries AR, Davies MN, Plomin R, Mill J: Allelic skewing of DNA methylation is widespread across the genome. Am J Hum Genet 2010;86:196-212.

14 Gertz J, Varley KE, Reddy TE, Bowling KM, Pauli F, Parker SL, et al: Analysis of DNA methylation in a three-generation family reveals widespread genetic influence on epigenetic regulation. PLoS Genet 2011;7:e1002228.

15 Zhang Y, Rohde C, Tierling S, Jurkowski TP, Bock C, Santacruz D, et al: DNA methylation analysis of chromosome 21 gene promoters at single base pair and single allele resolution. PLoS Genet 2009;5:e1000438.

16 Jablon S, Neel JV, Gershowitz H, Atkinson GF: The NAS-NRC twin panel: methods of construction of the panel, zygosity diagnosis, and proposed use. Am J Hum Genet 1967;19: 133-161.

17 Seddon JM, Sharma S, Adelman RA: Evaluation of the clinical age-related maculopathy staging system. Ophthalmology 2006;113: 260-266.
18 Klein R, Davis MD, Magli YL, Segal P, Klein BE, Hubbard L: The Wisconsin age-related maculopathy grading system. Ophthalmology 1991;98:1128-1134

19 Purcell S, Neale B, Todd-Brown K, Thomas L, Ferreira MAR, Bender D, et al: PLINK: a tool set for whole-genome association and population-based linkage analyses. Am J Hum Genet 2007;81:559-575.

20 Korn JM, Kuruvilla FG, McCarroll SA, Wysoker A, Nemesh J, Cawley S, et al: Integrated genotype calling and association analysis of SNPs, common copy number polymorphisms and rare CNVs. Nat Genet 2008;40: 1253-1260.

21 Neale BM, Fagerness J, Reynolds R, Sobrin L, Parker M, Raychaudhuri S, et al: Genomewide association study of advanced age-related macular degeneration identifies a role of the hepatic lipase gene (LIPC). Proc Natl Acad Sci USA 2010;107:7395-7400.

22 Iafrate AJ, Feuk L, Rivera MN, Listewnik ML, Donahoe PK, Qi Y, et al: Detection of largescale variation in the human genome. Nat Genet 2004;36:949-951.

23 International Schizophrenia Consortium: Rare chromosomal deletions and duplications increase risk of schizophrenia. Nature 2008;455:237-241.

24 Åberg K, Khachane AN, Rudolf G, Nerella S, Fugman DA, Tischfield JA, et al: Methylomewide comparison of human genomic DNA extracted from whole blood and from EBVtransformed lymphocyte cell lines. Eur J Hum Genet 2012;20:953-955.

25 Database of Single Nucleotide Polymorphisms (dbSNP). Bethesda, National Center for Biotechnology Information, National Library of Medicine (dbSNP Build ID:129).

26 Li LC, Dahiya R: MethPrimer: designing primers for methylation PCRs. Bioinformatics 2002;18:1427-1431. 
-27 Arányi T, Váradi A, Simon I, Tusnády GE: The BiSearch web server. BMC Bioinformatics 2006;7:431.

-28 Krueger F, Andrews SR: Bismark: a flexible aligner and methylation caller for BisulfiteSeq applications. Bioinformatics 2011;27: 1571-1572.

-29 Li H, Handsaker B, Wysoker A, Fennell T, Ruan J, Homer N, et al: The Sequence Alignment/Map format and SAMtools. Bioinformatics 2009;25:2078-2079.

- 30 Rachmilewitz J, Goshen R, Ariel I, Schneider T, de Groot N, Hochberg A: Parental imprinting of the human H19 gene. FEBS Lett 1992; 309:25-28.

-31 Williams CA, Zori RT, Stone JW, Gray BA, Cantu ES, Ostrer H: Maternal origin of 15q11-13 deletions in Angelman syndrome suggests a role for genomic imprinting. Am J Med Genet 1990;35:350-353.

- 32 Narayanan R, Butani V, Boyer DS, Atilano SR, Resende GP, Kim DS, et al: Complement factor $\mathrm{H}$ polymorphism in age-related macular degeneration. Ophthalmology 2007;114: 1327-1331.

- 33 Edwards AO, Ritter R, Abel KJ, Manning A, Panhuysen C, Farrer LA: Complement factor $\mathrm{H}$ polymorphism and age-related macular degeneration. Science 2005;308:421-424.

- 34 Haines JL, Hauser MA, Schmidt S, Scott WK, Olson LM, Gallins P, et al: Complement factor $\mathrm{H}$ variant increases the risk of age-related macular degeneration. Science 2005;308:419421.

- 35 Maller J, George S, Purcell S, Fagerness J, Altshuler D, Daly MJ, et al: Common variation in three genes, including a noncoding variant in $\mathrm{CFH}$, strongly influences risk of age-related macular degeneration. Nat Genet 2006;38 1055-1059.

-36 Fagerness JA, Maller JB, Neale BM, Reynolds RC, Daly MJ, Seddon JM: Variation near complement factor I is associated with risk of advanced AMD. Eur J Hum Genet 2009;17: 100-104.

-37 Gold B, Merriam JE, Zernant J, Hancox LS, Taiber AJ, Gehrs K, et al: Variation in factor $\mathrm{B}$ (BF) and complement component 2 (C2) genes is associated with age-related macular degeneration. Nat Genet 2006;38:458-462.

- 38 Yu Y, Bhangale TR, Fagerness J, Ripke S, Thorleifsson G, Tan PL, et al: Common variants near FRK/COL10A1 and VEGFA are associated with advanced age-related macular degeneration. Hum Mol Genet 2011;20: 3699-3709.

39 Rivera A, Fisher SA, Fritsche LG, Keilhauer $\mathrm{CN}$, Lichtner P, Meitinger T, Weber BH: Hypothetical LOC387715 is a second major susceptibility gene for age-related macular degeneration, contributing independently of complement factor $\mathrm{H}$ to disease risk. Hum Mol Genet 2005;14:3227-3236.
40 Dewan A, Liu M, Hartman S, Zhang SS, Liu DTL, Zhao C, et al: HTRA1 promoter polymorphism in wet age-related macular degeneration. Science 2006;314:989-992.

41 Maller JB, Fagerness JA, Reynolds RC, Neale BM, Daly MJ, Seddon JM: Variation in complement factor 3 is associated with risk of agerelated macular degeneration. Nat Genet 2007;39:1200-1201.

42 Yates JRW, Sepp T, Matharu BK, Khan JC, Thurlby DA, Shahid H, et al: Complement C3 variant and the risk of age-related macular degeneration. N Engl J Med 2007;357:553-561.

43 Chen W, Stambolian D, Edwards AO, Branham KE, Othman M, Jakobsdottir J, et al: Genetic variants near TIMP3 and high-density lipoprotein-associated loci influence susceptibility to age-related macular degeneration. Proc Natl Acad Sci USA 2010;107:7401-7406.

-44 Reimand J, Kull M, Peterson H, Hansen J, Vilo J: g:Profiler - a web-based toolset for functional profiling of gene lists from largescale experiments. Nucleic Acids Res 2007; 35:W193-200.

45 Javierre BM, Fernandez AF, Richter J, AlShahrour F, Martin-Subero JI, RodriguezUbreva J, et al: Changes in the pattern of DNA methylation associate with twin discordance in systemic lupus erythematosus. Genome Res 2010;20:170-179.

46 Dempster EL, Pidsley R, Schalkwyk LC, Owens S, Georgiades A, Kane F, et al: Diseaseassociated epigenetic changes in monozygotic twins discordant for schizophrenia and bipolar disorder. Hum Mol Genet 2011;20:47864796.

47 Wang X, Zhu H, Snieder H, Su S, Munn D, Harshfield G, et al: Obesity related methylation changes in DNA of peripheral blood leukocytes. BMC Med 2010;8:87.

48 Baranzini SE, Mudge J, van Velkinburgh JC, Khankhanian P, Khrebtukova I, Miller NA, et al: Genome, epigenome and RNA sequences of monozygotic twins discordant for multiple sclerosis. Nature 2010;464:1351-1356.

49 Stepanow S, Reichwald K, Huse K, Gausmann U, Nebel A, Rosenstiel P, et al: Allele-specific, age-dependent and BMI-associated DNA methylation of human MCHR1. PLoS One 2011;6:e17711.

50 Kang MY, Lee BB, Ji YI, Jung EH, Chun H-K, Song SY, et al: Association of interindividual differences in p14ARF promoter methylation with single nucleotide polymorphism in primary colorectal cancer. Cancer 2008;112: 1699-1707.

51 Boumber YA, Kondo Y, Chen X, Shen L, Guo Y, Tellez C, et al: An Sp1/Sp3 binding polymorphism confers methylation protection. PLoS Genet 2008;4:e1000162.
52 Wei L, Liu B, Tuo J, Shen D, Chen P, Li Z, et al: Hypomethylation of the IL17RC promoter associates with age-related macular degeneration. Cell Rep 2012;2:1151-1158.

53 Oliver VF, Franchina M, Jaffe AE, Branham KE, Othman M, Heckenlively JR, et al: Hypomethylation of the IL17RC promoterin peripheral blood leukocytes is not a hallmark of age-related macular degeneration. Cell Rep 2013;5:1527-1535.

54 Hunter A, Spechler PA, Cwanger A, Song Y, Zhang Z, Ying GS, et al: DNA methylation is associated with altered gene expression in AMD. Invest Ophthalmol Vis Sci 2012;53: 2089-2105.

55 Shoemaker R, Deng J, Wang W, Zhang K: Allele-specific methylation is prevalent and is contributed by CpG-SNPs in the human genome. Genome Res 2010;20:883-889.

- 56 Houseman E, Accomando WP, Koestler DC Christensen BC, Marsit CJ, Nelson HH, et al: DNA methylation arrays as surrogate measures of cell mixture distribution. BMC Bioinformatics 2012;13:86.

57 Glossop JR, Nixon NB, Emes RD, Haworth KE, Packham JC, Dawes PT, et al: Epigenomewide profiling identifies significant differences in DNA methylation between matchedpairs of T- and B-lymphocytes from healthy individuals. Epigenetics 2013;8:1188-1197.

58 Ramirez JM, Triviño A, Ramirez AI, Salazar JJ, Garcia-Sanchez J: Immunohistochemical study of human retinal astroglia. Vision Res 1994;34:1935-1946.

59 Pekny M, Nilsson M: Astrocyte activation and reactive gliosis. Glia 2005;50:427-434

60 Madigan MC, Penfold PL, Provis JM, Balind TK, Billson FA: Intermediate filament expression in human retinal macroglia. Histopathologic changes associated with age-related macular degeneration. Retina 1994;14:65-74.

61 Ramirez JM, Ramirez AI, Salazar JJ, de Hoz R, Triviño A: Changes of astrocytes in retinal ageing and age-related macular degeneration. Exp Eye Res 2001;73:601-615.

62 Wu KHC, Madigan MC, Billson FA, Penfold PL: Differential expression of GFAP in early v late AMD: a quantitative analysis. $\mathrm{Br} \mathrm{J}$ Ophthalmol 2003;87:1159-1166.

-63 Nikulina E, Tidwell JL, Dai HN, Bregman BS, Filbin MT: The phosphodiesterase inhibitor rolipram delivered after a spinal cord lesion promotes axonal regeneration and functional recovery. Proc Natl Acad Sci USA 2004;101: 8786-8790.

64 ENCODE Project Consortium: A user's guide to the encyclopedia of DNA elements (ENCODE). PLoS Biol 2011;9:e1001046.

65 Bell AC, Felsenfeld G: Methylation of a CTCF-dependent boundary controls imprinted expression of the Igf 2 gene. Nature 2000;405:482-485. 